\title{
REAL WORLD OCCLUSION CONSTRAINTS AND BINOCULAR RIVALRY
}

\author{
Shinsuke ShImojo* and Ken Nakayama \\ The Smith-Kettlewell Eye Research Institute, 2232 Webster St, San Francisco, CA 94115, U.S.A.
}

(Received 13 February 1989; in revised form 22 May 1989)

\begin{abstract}
A surface occluding a more distant surface gives rise to interocularly unpaired regions to its immediate left and right. The unpaired region on the left side is visible only to the left eye, whereas that on the right side is visible only to the right eye. Thus for real world scenes there are opto-geometrical constraints which determine whether particular combinations of relative depth and right-eye-only or left-eye-only stimuli are ecologically valid or invalid. We report a demonstration and experiments to show that opto-geometrically "valid" unpaired regions are seen as continuous with the rear plane and escape interocular suppression, whereas "invalid" unpaired regions are perceived as closer and are suppressed vigorously. An additionai experiment indicates that the results cannot be understood in terms of correspondence solving, but require neural mechanisms that embody real-world occlusion constraints. These results suggest a rather close interaction between stereopsis and rivalry "modules". Since explicit eye-of-origin information is lost relatively early in the hierarchical organization of cortical visual processing, we argue tha occlusion-related constraints must be embodied at such early levels.
\end{abstract}

$\begin{array}{lcccc}\begin{array}{l}\text { Rivalry } \\ \text { perception }\end{array} & \begin{array}{c}\text { Interocular suppression } \\ \text { Ecological optics }\end{array} & \text { Stereopsis } & \text { Interocular correlation } & \text { Binocular depth } \\ & & & \end{array}$

\section{INTRODUCTION}

We live in a 3-dimensional world, full of opaque objects and surfaces. This means that from any position in space, it is almost inevitable that some closer surface will occlude or hide more distant surfaces, either partially or entirely. Thus occlusion is perhaps one of the most obvious and fundamental "facts" about vision, a major physical obstacle that must be overcome by any viewer-centered system.

Recent studies in our laboratory indicate that the functional properties of early visual processing may be well suited to dealing with occlusion. For example, we have found that early visual processing related to depth and occlusion can assist in the pattern recognition of partially occluded objects (Nakayama, Shimojo \& Silverman, 1989) and can have a major role in the solution to the aperture problem for motion (Shimojo, Silverman \& Nakayama, 1989).

One of the interesting consequences of occlusion is that nearer objects occlude more distant objects to different extents in the two eyes. That is, there is always an interocularly-unpaired

"Current address: Department of Psychology, College of Arts and Sciences, University of Tokyo, Komaba, Meguro-ku, Tokyo 153, Japan. region contingent with vertically oriented surface discontinuities in natural scenes. The ecological "validity" of unpaired regions raises an intriguing question as to whether these regions are subject to binocular rivalry or suppression. Although much has been learned about interaction between inputs from the two eyes (see Wolfe, 1986 for a review), traditional studies of binocular rivalry and fusion have generally ignored such ecological issues and have concentrated on how the visual system deals with very different inputs in artificially created situations.

Our goal is to show evidence that such ecological analyses of natural scenes is relevant to human vision, and that occlusion related constraints are in fact implemented in the early visual processing.

\section{Occlusion and eye-of-origin constraints}

Figure 1 illustrates the specific problems and constraints that we explore in the current paper. When a flat square is seen in front of a more distant frontal-parallel surface or wall (left half of Fig. 1A), there are two narrow areas on the surface which are only visible monocularly, one on each side of the object (shadowed in the figure). Similarly, when the more distant surface is viewed through a rectangular window (left 

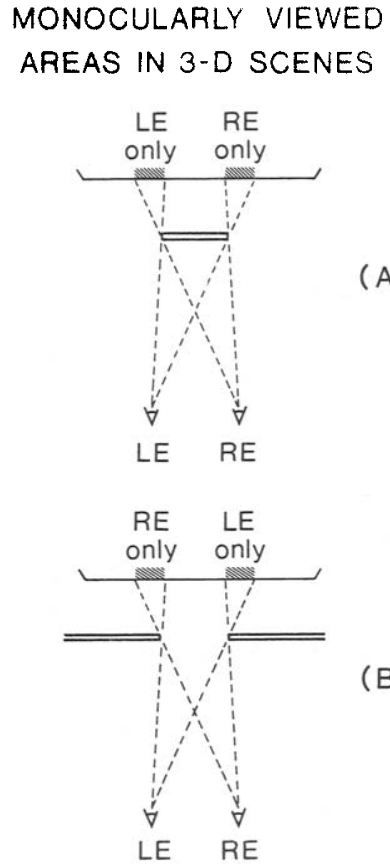

UNPAIRED AREAS IN RANDOM-DOT

STEREOGRAMS

(A)

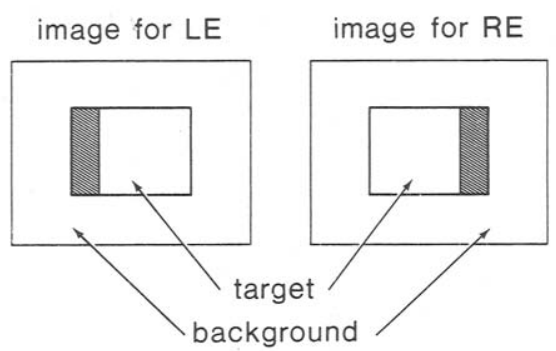

(B)

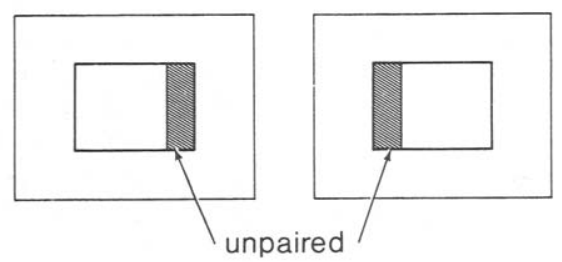

Fig. 1. A top view of interocularly-unpaired regions in natural 3-D scenes (left figure) and in random-dot stereograms (right figure). Interocularly-unpaired regions are indicated by shadows. (A) When the central rectangular region has a crossed disparity to the surround. (B) When the central region has uncrossed disparity.

half of Fig. 1B), there are also two areas on the surface which are seen by one eye only (shadowed in the figure). Note that unlike all other areas, these monocularly-viewed areas do not carry interocular disparity information.

Now, consider the artificial (experimental) counterparts of the situation just described. The right half of Fig. 1 illustrates interocularlyunpaired areas in random-dot stereograms for the object in front of a wall (Fig. 1A), or for the wall viewed through a window (Fig. 1B). When the stereogram having the central figure in crossed disparity (right half of Fig. 1A) is fused, the square region is perceived in front of the background, and the unpaired areas are clearly seen as belonging to the background (Lawson \& Gulick 1967; Julesz 1960, 1971). The perceived 3-dimensional structure is essentially the same as the physical structure illustrated in the left half of Fig. 1A.

When the stereogram with the uncrossed central region is fused (right half of Fig. 1B), the central region appears behind the surrounding foreground, as if it were a restricted view of another wall sitting behind the window. The unpaired areas are perceived as parts of the wall in back. Again, the perceived structure is the same as the real world counterpart which is illustrated in the left half of Fig. 1B. This time, the unpaired region on the right side, for instance, is given to the left eye, instead of right eye. But unpaired, or monocularly viewed areas are always perceived as continuous and seamless extensions of the more distant depth plane, regardless of the sign of disparity. Thus, perceptual depth localization is physically "reasonable", given that nearer objects occlude farther objects and that the amount of occlusion is different for the two eyes. The visual system apparently adopts a "more distant plane" interpretation for the unpaired areas, in spite of the lack of binocular correspondence and disparity.

The constraints can be summarized more clearly if we consider a single region of unpaired points in relation to the relative depth of adjacent surfaces. There are just four possibilities: two which are ecologically valid and two which are not. Figure 2 summarizes the constraints which are imposed by occlusion on the relationship between interocularly-paired and -unpaired visual inputs. When the front surface on the right side partially occludes the rear surface on the left side, as illustrated in Fig. 2A, the lateral distance between the two eyes allows the left eye to see more of the rear surface. Thus, visual 
(A)
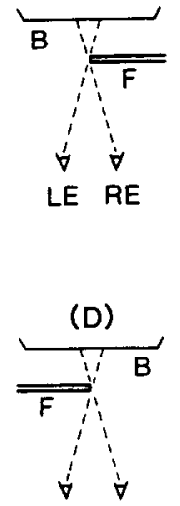

LE RE
(B)

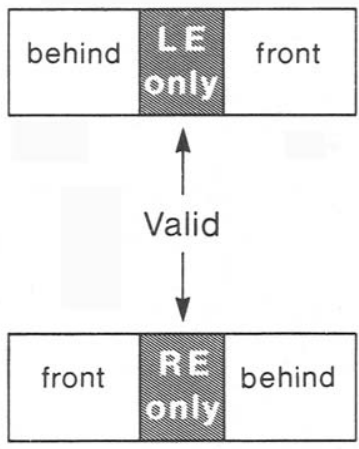

(E)
(C)

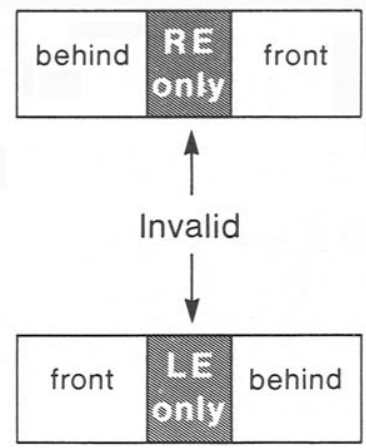

( $F)$

Fig. 2. Analysis of occlusion constraints. (A) A top view of the case where a front surface is on the right sicie. (B) Valid eye-of-origin of unpaired stimulus between the two surfaces in the case illustrated in (A). (C) Invalid eye-of-origin in the case (A). (D) A top view of the case where a front surface is on the left side. (E) Valid eye-of-origin in the case (D). (F) Invalid eye-of-origin in the case (D).

stimuli positioned between the two surfaces and visible only to left eye are opto-geometrically valid (Fig. 2B). The stimuli given only to right eye, however, should be considered impossible, or "invalid" (Fig. 2C) in the sense that there is no realistic physical structure which would yield such imputs to the eyes. Quite similarly, the stimuli given only to right eye would be valid if the surface on its left is in front and the surface on its right is behind (Fig. 2E) because this configuration and combination of stimuli is consistent with the real world situation where a front surface on the left side partially occludes a more distant surface on the right side (Fig. 2D). The stimuli given only to the left eye is "invalid" in this case (Fig. 2F).

\section{DEMONSTRATION: ASYMMETRIC DEPTH AND SUPPRESSION OF UNPAIRED REGIONS}

To see if the valid and the invalid cases of unpaired stimuli are processed differently, that is, to see if these occlusion-related constraints are in fact implemented in the human visual system, we developed a new demonstration. For reference, see Fig. 3. When appropriately fused (a cross fuser should fuse the left and the center images, whereas an uncrossed fuser should fuse

*The invalid case was generated by deleting a region which is of a size and shape comparable to the "valid" unpaired region, but on the opposite side of the central fusible disk in the same eye, and substituting it by homogeneous gray. the center and the right images), the two central black disks appear in front of the light-textured background. The curved, hatched areas (or "crescents") appear quite differently. Regarding the crescents next to the upper disk, the right one appears in front (at about the same depth as the disk) and has a tendency to fade away occasionally by interocular suppression whereas the left one appears as extension of the rear surface, and more stable with little sign of suppression. Note that these two crescentshaped patterns are both unpaired and comparable in the sense that neither has a corresponding part in the other eye. And yet, there is major differences in terms of depth localization and interocular suppression/rivalry. The only difference between the two crescents surrounding the upper disk is that the right crescent is "invalid" in terms of position and eye-of-origin (see Fig. 2F), whereas the left crescent is visible to the "valid" eye (see Fig. 2B).*

Comparable observations may be obtained from the crescents surrounding the lower disk. But this time, the left crescent appears in front and has a tendency to fade away; whereas the right crescent appears as an extension of the more distant surface, and is more stable without any notable sign of suppression. These observations are expected from the kind of analysis which is illustrated in Fig. 2: the left crescent is suppressed vigorously because it is "invalid" (Fig. 2C), and the right escapes from suppression because it is "valid" (Fig. 2E). By examin- 
ing the top and the bottom sets of stereogram, it should be clear. and important to note. that the observed asymmetric effects in depth and suppression can not be attributed to simple eye dominance because the "valid" and "invalid" stimuli are always given simultaneously to the same eye.

If a cross fuser fuses the center and the right images, or an uncrossed fuser fuses the left and the center images of Fig. 3, the central black disks will appear as behind the surrounding surface. Yet, comparable asymmetric effects will be observed, which are again consistent with and predicted from the real world occlusion layouts as described in Fig, 2.

\section{EXPERIMENT 1: ABSOLUTE JUDGMENT OF SUPPRESSION}

\section{Subjects and stimuli}

To assess these new findings more objectively and quantitatively, we ran 7 subjects (all naive except 1 subject) using stimuli, of which Fig. 4 illustrates an example. The stimulus consisted of a central square-shaped region, a background, and an interocularly-unpaired region, each of which was colored white, yellow and blue, respectively. This coloring arrangement was chosen to enhance the saliency of the rivalry between the different regions. Also, these regions were textured so that disparity cues of fusible regions would be enriched and that rivalry of unmatched areas between the two eyes would be facilitated.* The overall size of stimulus was $5.4 \times 5.9 \mathrm{deg}$, the central square extended $1.5 \times 2.8 \mathrm{deg}$, and the disparity of the width of unpaired region was $19 \mathrm{~min}$ arc at the observation distance of $85 \mathrm{~cm}$. The spaceaverage luminance of the center square region was approximately $110 \mathrm{~cd} / \mathrm{m}^{2}$. The central square was presented in either crossed or uncrossed disparity and seen in front or in back, respectively. The stimuius pattern for each eye was generated on a CRT screen (Commodore

* Our preliminary observations suggest that color and texture are not necessary conditions for the effect. however.

+Retinal correspondence changes depending on vergence. However, our pilot observations have indicated that the selective blockade of rivalry was independent of vergence or lateral shifts in fixation.
AMIGA 1000), and viewed by a prism haploscope.

\section{Eye-dominance test}

Before the main part of experiment, an eyedominance test was performed in which the stimuli were identical to those in Fig. 4 except that the interocular disparity of the central square was always zero (note that there is no such distinction between the valid and the invalid cases when there is no depth; or strictly speaking, the unpaired region is always invalid in the zero disparity condition). After a fusible frame presented for $1.5 \mathrm{sec}$, the stimulus was presented for a duration of $4.0 \mathrm{sec}$. The subject was asked to judge whether the blue (unpaired) area had faded even momentarily and to push one of two buttons accordingly. Four stimuli (2 eyes $\times 2$ sides) were presented 8 times each in a semi-randomized order (total of 32 trials). A $2 \times 2 \chi^{2}$-square test was applied to the data obtained from each subject, and the 3 subjects who reached a highly-significant level of eye dominance $(P<0.01)$ were eliminated from the main part of the experiment. (The justification was that if the subject suppresses one eye and not the other, the predictions of occlusion constraints could not be tested.) Thus, the remaining 7 subjects who had well-balanced binocularity participated in the main part of the experiment.

\section{Main experiment}

In the main part of experiment, eight possible stimuli ( 2 disparities $\times 2$ eyes $\times 2$ sides) were presented 8 times each in a semi-randomized order for a total of 64 trials. Again, the subject's task was to judge whether the blue, unpaired area had faded even if momentarily or partially. Durations of the fusible frame and the stimulus were identical to those in the eye dominance test. No fixation point was given, and subjects were allowed to observe the simulus freely. $\dagger$ As illustrated in Fig. 5. the results were straightforward: both in the crossed- and uncrosseddisparity conditions (Fig. 5A and B, respectively), all of the 7 subjects reported fading of the unpaired area more frequently in the invalid cases than in valid cases, except subject no. 6 in the crossed disparity condition where he reported no suppression at all $(P<0.05$ for the crossed, and $P<0.01$ for the uncrossed disparity condition). Furthermore, all of the 7 subjects who participated individually showed the significant difference by a $\chi^{2}$-square test 

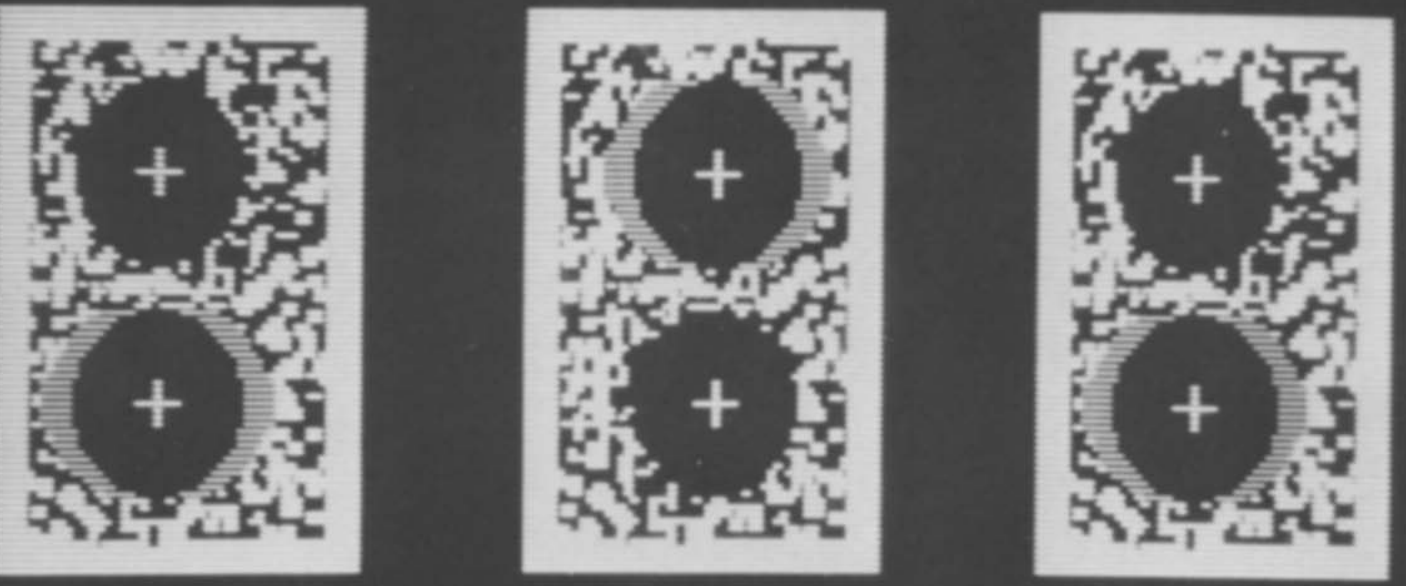

Fig. 3. "Unpaired crescent" demonstration. A cross fuser should fuse the left and the center images to perceive the central blank disk in front, and fuse the center and the right images to perceive the disk in the behind depth plane. An uncross fuser should do the opposite. Compare the gray crescents on both side of the disk in terms of depth and suppression. 


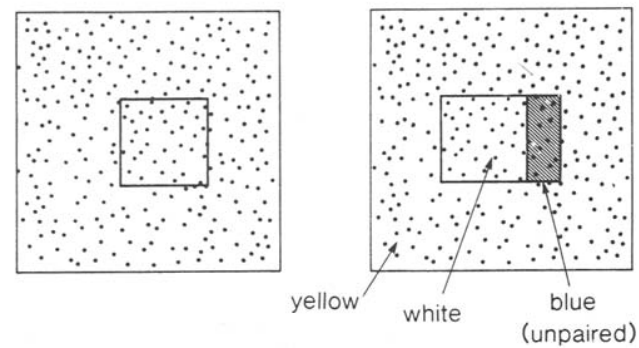

Fig. 4. Example of stimulus stereogram for expt 1.

$(P<0.01$ for 6 of them; $P<0.05$ for one of them).*

Note that since which eye is valid depends upon the depth relationship of the surrounding fusible areas (see Fig. 2), the valid eye changes depending on whether the unfused region is on the left or the right sides of the central square (see Fig. 1). Because the results from the two sides were categorized simply into the valid and invalid cases for the data analyses, eye dominance was counter-balanced, and can

* One could argue that different degree of rivalry between different combinations of colors might be the major cause of the obtained differences. For example, the blue, unpaired area corresponded to a part of the white central square in the other eye in the valid cases whereas it corresponded to a part of the yellow background in the other eye in the invalid cases, and this could potentially explain the higher rates of fading in the latter cases (a similar point can be made for Fig. 3). To test this possibility, 3 subjects who had participated in the original experiment were run again with new sets of stimuli in which the unpaired area was again colored blue, but the central square and the background switched their colors (so that color combinations were now reversed between the valid and the invalid cases). Note that if the combination of colors is the critical factor, then the rates of fading should be reversed between the valid and the invalid cases. The procedure for this control experiment was identical to the uriginal experiment. The obtained percentages of fading were as follows (data obtained in the original experiment are taken from Fig. 5 and shown in parentheses for a comparison):

\begin{tabular}{lcc} 
& \multicolumn{2}{c}{ Percentages of suppression > } \\
Valid & Invalid \\
Crossed & $13(11)$ & $67(54)$ \\
Uncrossed & $35(33)$ & $73(83)$
\end{tabular}

The newly obtained data were similar to the original data (Fig. 5). To test for statistical significance, the crossed and the uncrossed conditions were pooled together for each subject, and a $2 \times 2 \chi^{2}$-square test was applied. As a result, 2 out of the 3 subjects showed a highly significant difference between the valid and the invalid conditions, while one subject showed the same tendency which did not reach the statistically significant level. not explain the obtained difference in suppression rate between these two cases, either in the crossed-disparity or the uncrossed-disparity conditions.

Thus, our original observations (Fig. 3) were confirmed by an objective experiment with naive subjects. Interocularly-unpaired areas escape suppression if and only if their position relative to surrounding depth planes is in an optogeometrically valid relationship with respect to the eye which is stimulated.

\section{EXPERIMENT 2: MUTUAL UNPAIREDNESS HYPOTHESIS VS OCCLUSION CONSTRAINTS HYPOTHESIS}

The demonstration shown in Fig. 3 and the data obtained in the experiment reported above provide strong evidence for the importance of occlusion constraints in the suppression of binocularly unpaired points. One might still argue, however, that the asymmetric suppression effects that we have observed could be explained without referring to the real world complexity related to depth and occlusion. More specifically, a "mutual correlation" hypothesis has been suggested by Stuart Anstis (personal communication) as an alternative explanation of our data. This hypothesis makes
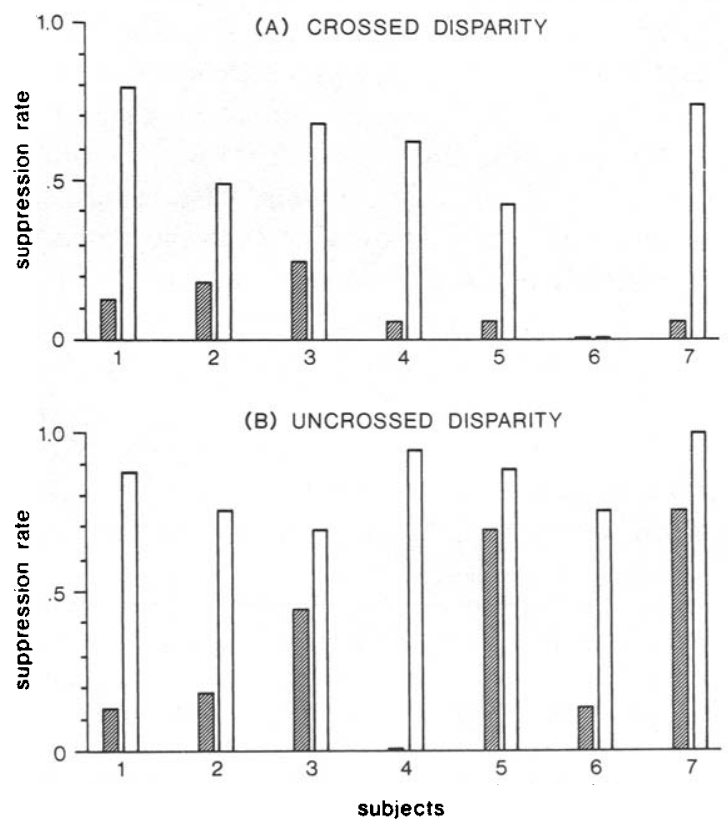

Fig. 5. Results of expt 1. Suppression rates were shown for both the valid cases (shadowed bars) and the invalid cases (white bars) for each subject. (A) Crossed-disparity condition. (B) Uncrossed-disparity condition of the central square. 
(A) HORIZONTAL DISPARITY

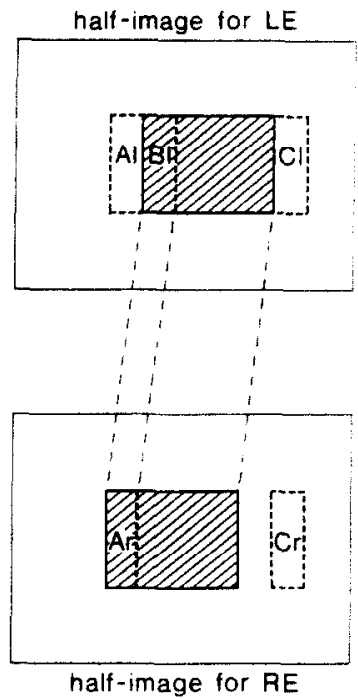

(B) VERTICAL DISPARITY

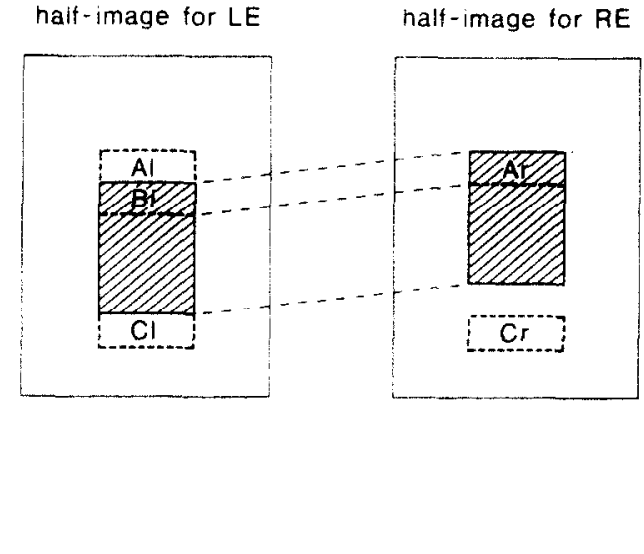

Fig. 6. Mutual unpairedness hypothesis. Retinally corresponding regions are denoted by $A, B$ and $C$. Eye is specified by subscripts $l$ and $r$. Dotted lines indicates correspondence with a disparity. (A) Horizontal disparity condition. (B) Vertical disparity condition.

the distinction between two forms of binocular unpairedness in the two eyes, one of which is assumed to be more rivalrous than the other. The basic thrust of the argument is that rivalry is not only dependent on the existence of monocularly-unpaired points, but it also depends on whether the retinotopic counterpart in the other eye are themselves paired or unpaired. More intense rivalry is predicted for the latter, mutually unpaired case presumably because of stronger competition between the eyes.

To illustrate this view, see Fig. 6A which compares the valid and invalid case in terms of their mutual correlation. For exarnple, region $A$ in the left eye $\left(A_{l}\right)$ has no counterpart in the right eye, yet its retinotopic counterpart (region $A$ in the right eye; $A_{r}$ ) does have a "mate" for fusion, it being region $B$ of the left eye $\left(B_{l}\right)$. So, the unpairedness seen in this case is only partial. Contrast this to the invalid case (region $C$ ) where each half image has no "mate" to be

*It has been known that vertical disparities are partly involved in binocular depth perception (the "induced effect": Ogle, 1950). However, that is not relevant here because we limit our discussion to foveal binocular vision, where there was no systematic relationship between the vertical disparity and perceived depth. (The fusible region at the center was usually perceived as slightly front or behind, as reported by Frisby \& Mayhew, 1978), but it was not reversed systematically by a reversal of the sign of disparity, unlike the case of horizontal disparity.) paired with in the other eye. Here the unpairedness is mutual, and one could argue that this condition could lead to more rivalry because the regions may be more "competitive". Although we are aware of no experimental or theoretical precedent to support this "mutual unpairedness" hypothesis, it is worth considering because this hypothesis offers an explanation for the results of the previous experiment without appealing to real world constraints related to depth and occlusion.

In order to evaluate the merits of this alternative hypothesis, a second experiment was conducted, based on the fact that the two hypotheses lead to grossly different sets of predictions when the disparity is vertical instead of horizontal. See Fig. 6B: our original hypothesis of opto-geometric or occlusion constraints would predict no difference between the two types of unpaired region in this case $\left(A_{r}\right.$ and $C_{r}$ in Fig. 6B), unlike in the case of horizontal disparity. The reason is that the orientation of disparity is now perpendicular to the axis which connects the two pupils. As such, there is no systematic relationship between depth in the 3-D environment and which eye is stimulated. In other words, there is no distinction such as geometrically "valid" vs "invalid" in the case of vertical disparity." The "mutual unpairedness" hypothesis, on the other hand, would still predict the existence of a difference in the two cases. $A_{l}$ may not be suppressed so much because $A_{r}$ is 
half-image for LE half-image for RE

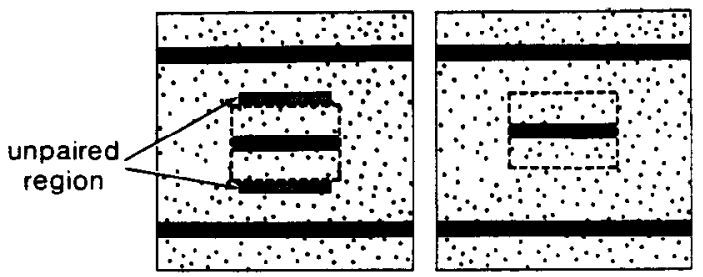

Fig. 7. Example of stimulus stereogram for expt 2.

paired with $B_{l}$, whereas $C_{l}$ may be suppressed vigorously because $C_{r}$ is not paired with any region in the right eye. (We will call $A_{l}$ a "non-competitive region", as opposed to $C_{l}$ as a "competitive region".) Unlike the occlusion hypothesis, it does not matter for the correspondence hypothesis whether the disparity is correlated with depth in the 3-D environment or whether the given unpaired region can be "valid" or "invalid".

\section{Subjects, stimuli and procedures}

To test these predictions, we ran 6 subjects. Though all of them participated in expt 1, 5 of them were still naive as to the purpose and specific predictions of the experiment. Each subject performed two sessions: one for the condition of horizontal disparity, the other for the condition of vertical disparity (always in this order). Unlike expt 1 where observers made a simple yes-no decision as to the existence of suppression, the present experiment employed a forced choice procedure. Two types of unpaired regions were given simultaneously on each side (left and right in the horizontal disparity condition, top and bottom in the vertical disparity condition) of the central fusible region in each stimulus in each eye, and the subject's task was to judge which of the two unpaired regions was more suppressed during the observation period of $9.0 \mathrm{sec}$. The amount of disparity, which is equal to the width of unpaired region, was identical for all conditions ( $8 \mathrm{~min}$ arc), and was much smaller than that in expt 1 . This smaller value was chosen in order to overcome the potential problem of double vision in the vertical disparity condition, and to make the horizontal and vertical disparity conditions comparable. (In fact, no subject reported double vision with this amount of vertical disparity when asked before the session.) Figure 7 illustrates an example of the stimuli. The overall size of stimulus was $5.2 \times 5.4 \mathrm{deg}$, with a central fusible region of $2.2 \times 2.3 \mathrm{deg}$ and the unpaired region at the observation distance of $85 \mathrm{~cm}$. The unpaired region was homogeneous and colored green, whereas both the central region and the background was colored red and textured. Averaged luminance was 80 and $60 \mathrm{~cd} / \mathrm{m}^{2}$, respectively. Thick, dark stripes (two in the background, one in the central region) were added, as shown in Fig. 7, to reinforce fusion and enrich disparity cues. Each of the four types of stimulus ( 2 eyes $\times 2$ signs of disparity) was repeated 12 times in a random order. Thus, each of the two sessions (one for the horizontal, the other for the vertical disparities) included 48 trials.

\section{Results and discussion}

Group data are shown in Fig. 8, where mean rates of suppression for the "invalid" or "competitive" region are presented for the two horizontal disparity conditions (crossed and uncrossed) and the vertical disparity condition. As obvious from the figure, the "invalid" or "competitive" side was suppressed more frequently in the two conditions of horizontal disparity, whereas no such bias from the chance level $(0.5)$ was found in the condition of vertical disparity.

Results of individual data analyses by $\chi^{2}$ square tests agreed with these tendencies in the group data. In the crossed disparity condition, the suppression rate of "invalid" side ranged from $58 \%$ to $100 \%$ across the 6 subjects. Thus, a tendency towards more suppression of the "invalid" region than the "valid" region was found in all of the subjects, of which 4 showed a statistically significant bias $(P<0.001$ for 3 , $P<0.05$ for 1 , and n.s. for 2 subjects). In the uncrossed disparity condition, the suppression rate of "invalid" side ranged from $54 \%$ to $96 \%$. Thus, a tendency towards more suppression of the "invalid" region than the "valid" region was found in all of the subjects, of which 4 showed statistically significant bias $(P<0.001$ for 3 , $P<0.05$ for 1 and NS for 2 subjects). In the vertical disparity condition, the suppression rate of "invalid" side ranged from $38 \%$ to $68 \%$. Three subjects showed a suppression rate larger than $50 \%$, two showed less than $50 \%$, and one showed $50 \%$ even. None of them significantly deviated from the chance level. Comparisons in individual subjects revealed that suppression of "competitive" side was reported more frequently in the crossed disparity than in the vertical disparity conditions in all 


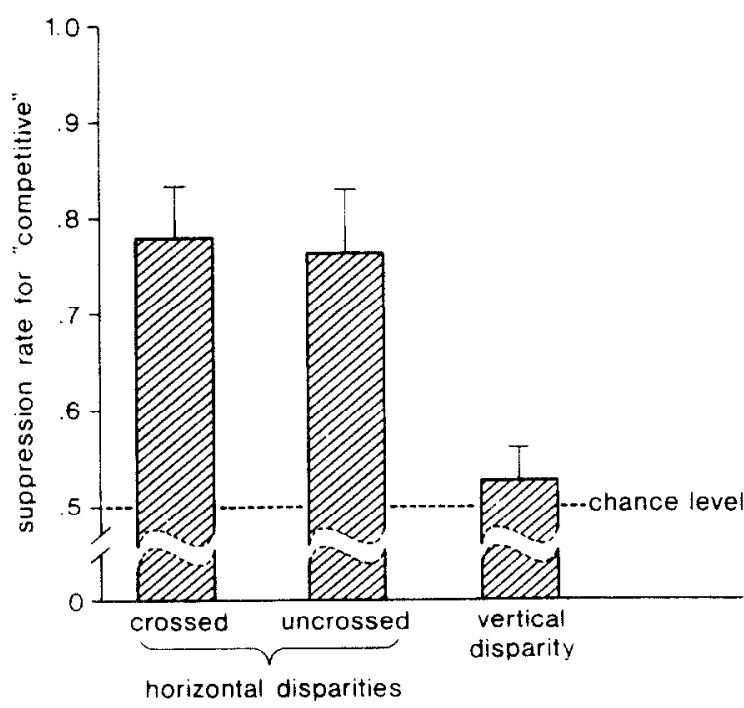

Fig. 8. Results of expt 2. Mean rates of suppression for the "competitive" side are shown for the crossed, the uncrossed, and the vertical disparity conditions.

the six subjects except one who showed tied scores, and more in the uncrossed disparity than in the vertical disparity conditions in all the subjects. However, a systematic bias was not found between the crossed and the uncrossed conditions ( 3 subjects showed one tendency, and the other 3 showed the opposite).

In short, the results of the expt 1 were confirmed with a much smaller horizontal disparity, in that the geometrically "valid" region is more free from suppression relative to the geometrically "invalid" region. Furthermore, the vertical disparity results argue strongly against a "mutual correlation" hypothesis. As such, it provides additional support for our view that opto-geometric contraints in the real world must be considered when addressing issues of rivalry and suppression.

\section{GENERAL DISCUSSION}

\section{Ecological relevancy of occlusion constraints}

Our results may be summarized in these two points:

(1) a region in a stereogram which was presented only to one eye escaped suppression when the monocular stimulation simulates the layout of surfaces in depth in the real world. When the monocular stimulation is presented to the optogeometrically invalid eye, however, it led to vigorous interocular suppression;
(2) the results cannot be explained by a difference in the process of correspondence solving, such as a difference in mutual unpairedness, because such an asymmetrical suppression effect was not observed in the experiment where the disparity was vertical and occlusion constraints were absent.

Interocularly-unpaired stimuli, either in random-dot correlograms or random-dot stereograms have been known to induce depth effects (O'Shea \& Blake, 1987; Mayhew \& Frisby, 1976; Frisby \& Mayhew, 1978; Holliday \& Braddick 1985; Collett, 1985; Julesz \& Chang, 1976). These reported effects however, do not really have direct relationship to the opto-geometric constraints of occlusion, and therefore, there is no real world correlate or interpretation. Unlike these studies, our phenomenological observation (Fig. 3) was that depth perception of interocularly unpaired region is different depending on whether the "valid" or the "invalid" eye was stimulated. Furthermore, the direction of this depth difference is consistent, at least partially, with predictions from opto-geometric analysis of occlusion (Kaye, 1978).

Close interaction between stereo and rivalry processes and their early cortical implementation

As early as 1961, Julesz noted that interocularly-unpaired regions in random-dot stereograms were perceived at the same depth as the background plane. He recognized the ecological validity of the depth perceived, but considered it a "highly central" phenomenon occurring after stereopsis (Julesz, 1960; 1971. pp. 259-261).

This raises the issue as to the level of processing required. At one extreme is the possibility that rivalrous perception of unpaired points is the consequence of higher order processes, "spatial reasoning" for example (Julesz, 1971: Rock, 1984). At the other extreme, and the one that we favor, is the possibility that it represents the outcome of autonomous neural processing, specific to vision and implemented very early in the visual pathway.

Our present results, taken together with anatomical and physiological studies of the primate visual system indicates that very early visual processing "takes account of" the occlusion constraints in the real world. Our most important firding is that rivalry is critically 
dependent on which eye receives the unpaired stimuli in relation to local depth signals. This establishes the close interaction between stereopsis and rivalry. In fact, another study from our laboratory suggests that unpaired stimuli alone can be a cue for the relative depth of neighboring surfaces (Nakayama \& Shimojo, 1990).

It also indicates that the processing must begin very early, sufficiently early in the stream of visual processing where the eye-of-origin information remains intact. This places it very close to area $\mathrm{V} 1$ or $\mathrm{V} 2$ because cells responding preferentially to one eye alone are built into the ocular dominance structure of $\mathrm{Vl}$ but are virtually absent in subsequent areas. Cells in area V2 and beyond are striking in their essentially equal responsiveness to either eye of stimulation (Burkhalter \& Van Essen, 1986; Maunsell \& Van Essen, 1983; Hubel \& Livingstone 1987). As such, these cells do not encode explicit eye-of-origin information (this early loss of eye-of-origin information may be reflected in the well known difficulty in the "utrocular perception"; Helmholtz, 1924; Smith, 1945). Thus, it seems more likely that the underlying mechanisms for the occlusion-related phenomenon seen in this paper includes cortical levels earlier than where eye-of-origin information is lost.

Further support for these views comes from related studies which also link occlusion constraints to eye-of-origin information. First, we simulated the viewing of a small horizontally moving target behind a narrow vertical slit (Shimojo, Silverman \& Nakayama, 1988). This situation precludes ordinary stereopsis because the target is not visible simultaneously in the right and left eye, yet single vision and appropriate depth were seen for physically valid combinations of motion direction and interocular sequence. Invalid combinations, on the other hand, were seen as unfused with degenerate depth.

We also found a special case of subjective contour formation which is also closely related to the occlusion constraints: only one or two unpaired dots are sufficient to form a subjective edge, positioned so that it is consistent with an occluding edge, which in the real world would block stimulation to one eye while letting them be visible to the other (Nakayama \& Shimojo, 1988; 1990). Our current findings, taken together with these phenomena, underscore the important roles of interocularly- unpaired stimuli and eye-or-origin information in depth processing. As mentioned earlier, visual neurons are non-selective to eye stimulated in the higher cortical levels (Burkhalter \& Van Essen, 1986; Maunsell \& Van Essen, 1983), leading us to hypothesize that occlusion constraints based on eye-of-origin information are implemented at relatively early levels of cortical processing, possibly as early as V1.

Acknowledgements - The current research is partially supported by NIH grant EY06522, and by AFOSR grant 83-0320. Shinsuke Shimojo is supported by Fellowship of the Japanese Society for the Promotion of Science for Japanese Junior Scientists, and by a Rachel C. Atkinson Fellowship.

\section{REFERENCES}

Burkhalter, A. \& Van Essen, D. C. (1986). Processing of color, form and disparity information in visual areas VP and $\mathrm{C} 2$ of ventral extrastriate cortex in the macaque monkey. Journal of Neuroscience, 6, 2327-2351.

Collett, T. S. (1985). Extrapolating and interpolating surfaces in depth. Proceedings of the Royal Society of London, Series B, Biological Sciences, 224, 43-56.

Frisby, J. P. \& Mayhew, J. E. W. (1978). The relationship between apparent depth and disparity in rivalrous-texture stereograms. Perception, 7, 661-678.

Helmholtz H. V. (1924). Treatise on physiological optics (Translated by Southall, J. P. C.). New York: (Original work published in 1909).

Holliday, I. E. \& Braddick, O. J. (1985). Depth perception of uncorrelated areas in random-dot stereograms. Perception, 14, A25-26.

Hubel, D. H. \& Livingstone, M. S. (1987). Segregation of form, color and stereopsis in primate area 18. Journal of Neuroscience, 7, 3378-3415.

Julesz, B. (1960). Binocular depth perception of computergenerated patterns. Bell System Technical Journal, 29, $1125-1162$.

Julesz. B. (1971). Foundation of cyclopean perception. Chicago: University of Chicago Press.

Julesz, B. \& Chang, J. (1976). Interaction between pools of binocular disparity detectors tuned to different disparities. Biological Cybernetics, 22, 107-119.

Kaye, M. (1978). Stereopsis without binocular correlation. Vision Research, 18, 1013-1022.

Lawson, R. B. \& Gulick, W. L. (1967). Stereopsis and anomalous contour. Vision Research, 7, 271-297.

Maunsell, J. H. R. \& Van Essen, D. C. (1983). Functional properties of neurons in middle temporal visual area of the macaque monkey. I. Selectivity for stimulus direction, speed, and orientation. Journal of Neurophysiology, 49, 1148-1167.

Mayhew, J. E. W. \& Frisby, J. P. (1976). Rivalrous texture stereograms. Nature, London, 264, 53-56.

Nakayama, K. \& Shimojo, S. (1988). Depth, rivalry and subjective contours from unpaired monocular points. Investigative Ophthalmology and Visual Science, Annual Meeting Abstracts Issue, p. 21.

Nakayama, K. \& Shimojo, S. (1990). DaVinci stereopsis: Depth and subjective contours from unpaired points. Vision Research (submitted). 
Nakayama, K., Shimojo, S. \& Silverman. G. H. (1989). Stereoscopic depth. Its relation to image segmentation, grouping, and the recognition of occluded objects. Perception, 18, 55-68.

Ogle, K. N. (1950). Research in binocular vision. Philadelphia: Saunders.

O'Shea, R. P. \& Blake, R. (1987). Depth without disparity in random-dot stereograms. Perception and Psychophysics, 42, 205-214.

Rock, I. (1984). Perception. New York: Scientific American Books.
Shimojo. S., Silverman. G. H. \& Nakayama, K. (1988). An occlusion-related mechanism of depth perception based on motion and interocular sequence. Nature. London. 33.7 . $265 \div 268$.

Shimojo, S., Silverman, G. H. \& Nakayama, K. (1989). Occlusion and the solution to the aperture problem for motion. Vision Research, 29, 619-626.

Smith. S. (1945). Utrocular or "which eye" discrimination. Journal of Experimental Psychology, 35. 1-14.

Wolfe. J M. (1986). Stereopsis and binocular rivalry. Psichological Review: 93. 269-282. 